\title{
Topological/Geometric Properties of the Orbit Accumulation Set 12
}

\author{
Steven G. Krantz 3
}

September 25, 2018

\begin{abstract}
We study the set of boundary orbit accumulation points of the automorphism group action on a bounded domain in $\mathbb{C}^{n}$. Topological and geometric properties of this set are derived.
\end{abstract}

\section{Introduction}

A domain $\Omega$ in $\mathbb{C}^{n}$ is a connected, open set. An automorphism of $\Omega$ is a biholomorphic self-map. The collection of automorphisms forms a group under the binary operation of composition of mappings. The topology on this group is uniform convergence on compact sets, or the compact-open topology. We denote the automorphism group by Aut $(\Omega)$.

Although domains with transitive automorphism group are of some interest, they are relatively rare (see [HEL]). A geometrically more natural condition to consider, and one that gives rise to a more robust and broader class of domains, is that of having non-compact automorphism group. Clearly a domain has non-compact automorphism group if there are automorphisms

\footnotetext{
${ }^{1}$ Key Words: orbit accumulation set, automorphism, orbit, holomorphic mapping.

${ }^{2}$ MR Classification Numbers: 32M05,32M99.

${ }^{3}$ Author supported in part by the National Science Foundation and by the Dean of the Graduate School at Washington University.
} 
$\left\{\varphi_{j}\right\}$ which have no subsequence that converges to an automorphism. The following proposition of Henri Cartan is of particular utility in the study of these domains:

Proposition 0.1 Let $\Omega \beta \mathbb{C}^{n}$ be a bounded domain. Then $\Omega$ has non-compact automorphism group if and only if there are a point $X \in \Omega$, a point $P \in \partial \Omega$, and automorphisms $\varphi_{j}$ of $\Omega$ such that $\varphi_{j}(X) \rightarrow P$ as $j \rightarrow \infty$.

We refer the reader to [NAR] for discussion and proof of Cartan's result.

A point $P$ in $\partial \Omega$ is called a boundary orbit accumulation point if there is an $X \in \Omega$ and automorphisms $\varphi_{j}$ of $\Omega$ such that $\lim _{j \rightarrow \infty} \varphi_{j}(X)=P$.

The inspiration for the present paper comes from [ISK1]. In that paper, we considered the set $S(\Omega)$ of all boundary orbit accumulation points of a smoothly bounded, finite type domain $\Omega$ in $\mathbb{C}^{n}$ (see [KRA1] for the concept of finite type). In particular, we showed that, if the set $S$ contains at least three points, then it is perfect. Also, if $S$ is not connected, then it has uncountably many connected components. Finally, the set $S$ is generically contained in the set where the Levi form has minimal rank and where the type and the multiplicity functions are maximal.

Our goal in this paper is to extend and generalize these results. We also provide examples to show that some of these statements are sharp.

\section{Some Examples}

We begin with some examples. These will all be smooth deformations of the unit ball in $\mathbb{C}^{2}$.

EXAMPLE 1.1 There is a bounded domain $\Omega$ in $\mathbb{C}^{2}$ with Lipschitz boundary with the property that $S(\Omega)$ has just two points.

To see this, let $B$ be the unit ball in $\mathbb{C}^{2}$. For $j \in \mathbb{Z}$, let

$$
\varphi\left(z_{1}, z_{2}\right)=\left(\frac{z_{1}-1 / 5}{1-(1 / 5) z_{1}}, \frac{\sqrt{1-(1 / 5)^{2}} z_{2}}{1-(1 / 5) z_{1}}\right)
$$

and let $\varphi^{j}$ denote the composition of $\varphi$ with itself $j$ times (and of course $\varphi^{j}=\left(\varphi^{-1}\right)^{|j|}$ when $j<0$ and $\left.\varphi^{0}=\mathrm{id}\right)$.

Then each $\varphi^{j}$ is an automorphism of $B$. Furthermore,

$$
\lim _{j \rightarrow+\infty} \varphi^{j}\left(z_{1}, z_{2}\right)=(-1,0)
$$


uniformly on compact subsets of $\left(z_{1}, z_{2}\right)$ in $B$. And

$$
\lim _{j \rightarrow-\infty} \varphi^{j}\left(z_{1}, z_{2}\right)=(1,0)
$$

uniformly on compact subsets of $\left(z_{1}, z_{2}\right)$ in $B$.

Now define

$$
\alpha\left(z_{1}, z_{2}\right)=\left\{\begin{array}{lll}
\left(1 / 100-\left|z_{1}\right|^{2}\right)^{2} \cdot\left(1 / 100-\left|z_{2}\right|^{2}\right)^{2} & \text { if } \quad\left|\left(z_{1}, z_{2}\right)\right| \leq 1 / 10 \\
0 & \text { if }\left|\left(z_{1}, z_{2}\right)\right|>1 / 10
\end{array}\right.
$$

We set

$$
\Omega^{\prime}=\left\{\left(z_{1}, z_{2}\right) \in \mathbb{C}^{2}:\left(\left|z_{1}\right|^{2}+\left|z_{2}\right|^{2}-1\right)+\alpha\left(z_{1}-i, z_{2}\right)<0\right\} .
$$

Finally, let

$$
\Omega=\bigcap_{j=-\infty}^{\infty} \varphi^{j}\left(\Omega^{\prime}\right)
$$

We see that $\Omega$ is the unit ball with infinitely many smooth dents which accumulate at $(1,0)$ and $(-1,0)$. Moreover, the automorphism group of $\Omega$ is just the cyclic group $\left\{\varphi^{j}\right\}$ (see [LER], [GRK2] for the details).

EXAMPLE 1.2 There is a bounded domain $\Omega$ in $\mathbb{C}^{2}$ with Lipschitz boundary with the property that $S(\Omega)$ has just one point.

To see this, let $B$ be the unit ball in $\mathbb{C}^{2}$. For $j \in \mathbb{Z}$, let

$$
\varphi^{j}\left(z_{1}, z_{2}\right)=\left(\frac{2 i-j}{2 i+j} \cdot \frac{z_{1}+j /(j-2 i)}{1+z_{1} j /(j+2 i)}, \frac{2 i z_{2} /(j+2 i)}{1+z_{1} j /(j+2 i)}\right) .
$$

The collection $\left\{\varphi^{j}\right\}_{j=-\infty}^{\infty}$ forms a cyclic subgroup of the automorphism group of $B$. One may see this by mapping $B$ to the Siegel upper half plane $\mathcal{U}=$ $\left\{\left(w_{1}, w_{2}\right): \operatorname{Im} w_{1}>\left|w_{2}\right|^{2}\right\}$ and transferring the automorphims to $\mathcal{U}$ (see [KRA2] for the details of this process). In fact, on $\mathcal{U}$, the automorphisms $\varphi^{j}$ become real translations in the $w_{1}$ variable.

Then

$$
\lim _{j \rightarrow \pm \infty} \varphi^{j}\left(z_{1}, z_{2}\right)=(-1,0)
$$

uniformly on compact subsets of $\left(z_{1}, z_{2}\right)$ in $B$. 


\section{Examples Based on the Bidisc}

The examples in this section are certainly of some utility, but they are ancillary to the main points we are trying to make in this paper. We include them for completeness and for interest's sake.

EXAMPLE 2.1 There is a domain $\Omega \beta \mathbb{C}^{n}$, modeled on the bidisc $D^{2}$, with the property that $S(D)$ has dimension 0 .

EXAMPLE 2.2 There is a domain $\Omega ß \mathbb{C}^{n}$, modeled on the bidisc $D^{2}$, with the property that $S(D)$ has dimension 1 .

EXAMPLE 2.3 There is a domain $\Omega ß \mathbb{C}^{n}$, modeled on the bidisc $D^{2}$, with the property that $S(D)$ has dimension 2 .

EXAMPLE 2.4 There is a domain $\Omega ß \mathbb{C}^{n}$, modeled on the bidisc $D^{2}$, with the property that $S(D)$ has dimension 3 .

Discussion of Examples 2.1-2.4: Let $P_{0} \in \partial D^{2}$ be given by $P_{0}=(i, i)$. Let

$$
\varphi\left(z_{1}, z_{2}\right)=\left(\frac{z_{1}+1 / 5}{1+(1 / 5) z_{1}}, \frac{z_{2}+1 / 5}{1+(1 / 5) z_{2}}\right) .
$$

As usual, let $\varphi^{j}$ be the composition of $\varphi$ with itself $j$ times. Then

$$
\lim _{j \rightarrow \pm \infty} \varphi^{j}\left(P_{0}\right)=( \pm 1, \pm 1) .
$$

If we set up a "dent" at $P_{0}$, just as in Examples 1.1 and 1.2, and propagate the dent using the automorphisms $\varphi^{j}$, then we produce a domain with automorphism group a cyclic group and two orbit accumulation points. This is a zero-dimensional $S(\Omega)$.

Now let us modify the discussion in the preceding paragraph by defining the disc automorphism

$$
\lambda(\zeta)=\frac{\zeta+1 / 5}{1+(1 / 5) \zeta}
$$

and then considering the automorphisms

$$
\psi_{j, \rho}\left(z_{1}, z_{2}\right)=\left(\lambda^{j}\left(z_{1}\right), \rho^{j}\left(z_{2}\right)\right)
$$

where $\lambda^{j}$ as usual denote the $j$-fold composition of $\lambda$ with itself and $\rho$ denotes any nontrivial Möbius transformation $\zeta \mapsto(\zeta-a) /(1-\bar{a} \zeta)$ of the disc $D$. 
As usual, we construct a small dent at the boundary point $(i, 0)$ and we propagate it using the automorphisms $\psi_{j, \rho}$ for all integers $j$ and all automorphisms $\rho$ of the unit disc. Then it is easy to see that the boundary orbit accumulation points of the resulting domain $\Omega$ are points of the form

$$
\{1\} \times \partial D \quad \text { and } \quad\{-1\} \times \partial D .
$$

We see that, according to this construction, the dimension of $S(\Omega)$ is 1 .

Now we modify the construction in the last two paragraphs by letting

$$
\mu_{j}\left(z_{1}, z_{2}\right)=\left(\lambda^{j}\left(z_{1}\right), z_{2}\right)
$$

We construct a domain with dents as usual - a modification of the bidisc $D^{2}$ - using these automorphisms. It is easy to see that, for the resulting $\Omega$, the set $S(\Omega)$ is

$$
(\{-1\} \times D) \cup(\{1\} \times D) .
$$

Thus $S(D)$ is of dimension 2 .

Finally, if we consider just the usual bidisc $D^{2}$ with its full automorphism group, then the set $S\left(D^{2}\right)$ is the entire topological boundary $\partial D \times D \cup D \times \partial D$. Thus $S\left(D^{2}\right)$ has dimension 3 .

\section{General Principles}

Now we wish to make some general assertions about the geometric nature of the orbit accumulation set $S(\Omega)$.

Proposition 3.1 Let $\Omega ß \mathbb{C}^{n}$ be a smoothly bounded domain of finite type in the sense of Catlin/D'Angelo/Kohn (see [KRA1] for details). Then it is impossible for $S(\Omega)$ to contain a relatively open subset $U$ of $\partial \Omega$ unless $\Omega$ is biholomorphic to the unit ball $B$.

Proof: This assertion is particularly easy to see in complex dimension 2 . For if a relatively open subset $U$ of the boundary has all points of geometric type greater than 2, then $U$ is foliated by 1-dimensional complex analytic varieties (see [FRE]) 4 So every point of $U$ is in fact of infinite type and that is a contradiction. Thus any relatively open subset $U$ of $\partial \Omega$ will contain

\footnotetext{
${ }^{4}$ One could at this point invoke the theorem of Kim $[\mathrm{KIM}]$ - at least in the convex case - to see that $\Omega$ must be biholomorphic to the bidisc. That would be a contradiction.
} 
points of type 2. Of course these points must be pseudoconvex by the result of [GRK1]. Hence the points are strongly pseudoconvex. But then the theorem of Bun Wong and Rosay (see [WON] and [ROS]) tells us that the domain $\Omega$ is biholomorphic to the unit ball and hence $S(\Omega)$ is the entire sphere. That proves the result.

In $\mathbb{C}^{n}$ with $n>1$ we must instead use the structure theorem of Catlin $[\mathrm{CAT}]$ which tells us that the set of finite type points with the type greater than 2 forms a subset of the boundary of codimension at least 1 . Hence any relatively open subset of the boundary will contain pseudoconvex points of type 2 , and the argument is finished as before.

Proposition 3.2 Let $\Omega \AA \mathbb{C}^{n}$ be a smoothly bounded domain of finite type in the sense of Catlin/D'Angelo/Kohn (see [KRA1] for details). Then it is impossible for $S(\Omega)$ to contain a subset $E$ of $\partial \Omega$ having positive $(2 n-1)$ dimensional Hausdorff measure unless $\Omega$ is biholomorphic to the unit ball $B$.

Proof: Reasoning as in the second part of the proof of the preceding proposition, we see that the set $E$ must contain a strongly pseudoconvex point. It follows then from the Bun Wong/Rosay theorem that the domain must be biholomorphic to the unit ball.

\section{Closing Thoughts}

There is no Riemann mapping theorem in several complex variables. So we seek other means to compare and contrast domains up to biholomorphic equivalence. Certainly the automorphism group is one invariant that has proved to be both flexible and useful.

One avenue that has been explored to a great extent is the Levi geometry of boundary orbit accumulation points (see [GRK3] and [ISK2]). The nature of the boundary orbit accumulation set is a much newer avenue of inquiry, and there remain many questions to be answered in this venue. We hope to explore some of them in future papers. 


\section{REFERENCES}

[BEB] S. Bell and H. Boas, Regularity of the Bergman projection in weakly pseudoconvex domains, Math. Ann. 257(1981), 23-30.

[CAT] D. Catlin, Boundary invariants of pseudoconvex domains, Ann. of Math. 120(1984), 529-586

[CHM] S.-S. Chern and J. Moser, Real hypersurfaces in complex manifolds, Acta Math. 133(1974), 219-271.

[FRE] M. Freeman, Local complex foliation of real submanifolds, Math. Ann. 209(1974), 1-30.

[GRK1] R. E. Greene and S. G. Krantz, Techniques for studying automorphisms of weakly pseudoconvex domains, Several Complex Variables (Stockholm, 1987/1988), Math. Notes 38, Princeton University Press, Princeton, NJ, 1993, 389-410.

[GRK2] R. E. Greene and S. G. Krantz, Invariants of Bergman geometry and the automorphism groups of domains in $\mathbb{C}^{n}$, Geometrical and Algebraical Aspects in Several Complex Variables (Cetraro, 1989), 107-136, Sem. Conf., 8, EditEl, Rende, 1991.

[GRK3] R. E. Greene and S. G. Krantz, Biholomorphic self-maps of domains, Complex Analysis II (C. Berenstein, ed.), Springer Lecture Notes, vol. 1276, 1987, 136-207.

[HEL] S. Helgason, Differential Geometry and Symmetric Spaces, Academic Press, New York, 1962.

[ISK1] A. Isaev and S. G. Krantz, On the boundary orbit accumulation set for a domain with non-compact automorphism group, Mich. Math. Jour. 43(1996), 611-617.

[ISK2] A. Isaev and S. G. Krantz, Domains with non-compact automorphism group: a survey, Adv. Math. 146(1999), 1-38.

[KIM] K.-T. Kim, Domains in $\mathbb{C}^{n}$ with a piecewise Levi flat boundary which possess a noncompact automorphism group, Math. Ann. 292(1992), $575-586$. 
[KIK] K.-T. Kim and S. G. Krantz, Complex scaling and domains with noncompact automorphism group, Illinois Journal of Math. 45(2001), 1273-1299.

[KRA1] S. G. Krantz, Function Theory of Several Complex Variables, $2^{\text {nd }}$ ed., American Mathematical Society, Providence, RI, 2001.

[NAR] R. Narasimhan, Several Complex Variables, University of Chicago Press, Chicago, 1971.

[KRA2] S. G. Krantz, Explorations in Harmonic Analysis, with Applications in Complex Function Theory and the Heisenberg Group, Birkhäuser Publishing, Boston, 2009.

[LER] L. Lempert and L. A. Rubel, An independence result in several complex variables, Proc. Amer. Math. Soc. 113(1991), 1055-1065.

[NAR] R. Narasimhan, Several Complex Variables, University of Chicago Press, Chicago, 1971.

[ROS] J.-P. Rosay, Sur une characterization de la boule parmi les domains de $\mathbb{C}^{n}$ par son groupe d'automorphismes, Ann. Inst. Four. Grenoble XXIX(1979), 91-97.

[WON] B. Wong, Characterizations of the ball in $\mathbb{C}^{n}$ by its automorphism group, Invent. Math. 41(1977), 253-257.

Department of Mathematics

Washington University in St. Louis

St. Louis, Missouri 63130

sk@math.wustl.edu 